\title{
HAUSDORFF AND BOX DIMENSIONS OF CONTINUOUS FUNCTIONS AND LINEABILITY
}

\author{
A. BONILLA, G.A. MUÑOZ-FERNÁNDEZ, J.A. PRADO-BASSAS, \\ AND J.B. SEOANE-SEPÚLVEDA
}

\begin{abstract}
Given $s \in(1,2]$, we study (among other questions) the algebraic genericity of the set of continuous functions $f:[0,1] \rightarrow \mathbb{R}$ whose graph has Hausdorff (or Box) dimension exactly $s$.
\end{abstract}

\section{INTRODUCTION AND PRELIMINARIES}

It is a classical conjecture of Benoît B. Mandelbrot from 1977 (see [17]) that the Hausdorff dimension of the graph of the Weierstrass function

$$
W_{\lambda, b}(x)=\sum_{n=0}^{\infty} \lambda^{n} \cos \left(2 \pi b^{n} x\right) \quad x \in \mathbb{R}
$$

is equal to

$$
D=2+\frac{\log \lambda}{\log b}
$$

where $b \in \mathbb{N}, 0<\lambda<1<b$ and $\lambda b>1$. Many authors have worked in this problem for over 30 years. This conjecture has been proved recently by W. Shen ([21], see also [16]). In fact, due to the periodicity of the Weierstrass function, its Hausdorff dimension is the same if it is restricted to $[0,1]$.

Given a function $f: A \subset \mathbb{R} \rightarrow \mathbb{R}$, by $\operatorname{graph}(f)$ we shall denote the graph of this function, that is,

$$
\operatorname{graph}(f)=\{(x, f(x)), x \in A\} \subset \mathbb{R}^{2} .
$$

If $B \subset A$, by $\operatorname{graph}_{B}(f)$ we mean the set $\{(x, f(x)), x \in B\}$, that is, the part of $\operatorname{graph}(f)$ supported on $B$.

In order to make this article self-contained, we now include the definitions of Hausdorff and Box dimensions.

Given a set $F \subset \mathbb{R}^{n}$, the diameter of $F$ is $|F|:=\sup \{|x-y|: x, y \in F\}$. Given $\delta>0$, a $\delta$-cover of $F$ is a countable collection $\left(U_{i}\right)_{i}$ of subsets of $\mathbb{R}^{n}$ such that $F \subset \bigcup_{i} U_{i}$ and $\left|U_{i}\right|<\delta$ for all $i$.

2010 Mathematics Subject Classification. 11K55, $15 \mathrm{~A} 03$.

Key words and phrases. lineability, spaceability, algebrability, Hausdorff dimension, Box dimension.

A. Bonilla was supported by MINECO MTM2016-75963-P. J.A. Prado-Bassas was supported by the Plan Andaluz de Investigación de la Junta de Andalucía FQM-127 Grant P08-FQM-03543 and by MINECO Grant MTM2015-65242-C2-1-P. G.A. MuñozFernández and J.B. Seoane-Sepúlveda were supported by Grant MTM2015-65825-P. 


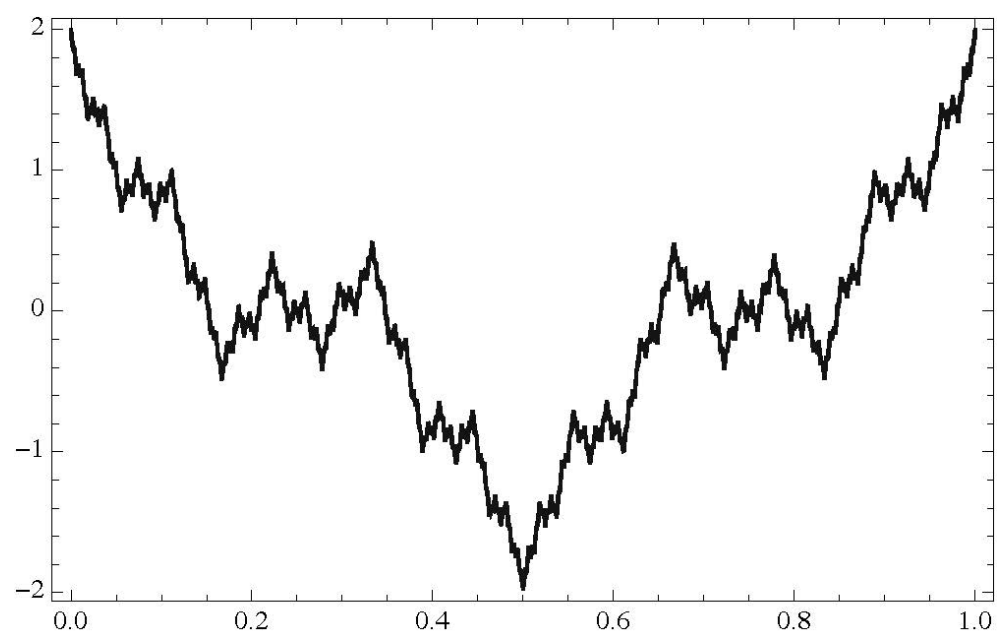

Figure 1. A sketch of the famous Weierstrass' Monster (for $\lambda=1 / 2$ and $b=3$ ), of Hausdorff dimension equals to $2-$ $\frac{\log (2)}{\log (3)} \approx 1.3690702464$.

For any subset $M$ of $\mathbb{R}^{N}$ and any $\delta>0$, let

$$
\mathcal{H}_{\delta}^{s}(M):=\inf \left\{\sum_{i=1}^{\infty}\left|U_{i}\right|^{s}:\left(U_{i}\right)_{i} \text { is a } \delta \text { - cover of } M\right\},
$$

and write

$$
\mathcal{H}^{s}(M)=\lim _{\delta \rightarrow 0} \mathcal{H}_{\delta}^{s}(M)
$$

The Hausdorff dimension of $M$ is

$$
\operatorname{dim}_{H}(M):=\inf \left\{s \geq 0: \mathcal{H}^{s}(M)=0\right\}=\sup \left\{s \geq 0: \mathcal{H}^{s}(M)=+\infty\right\} .
$$

Furthermore, given any non-empty subset $M$ of $\mathbb{R}^{n}$, let $N_{\delta}(M)$ denote the smallest number of sets, of diameter at most $\delta$, needed to cover $M$. Then the lower and upper box dimensions of $M$ are

$$
\underline{\operatorname{dim}_{B}}(M):=\liminf _{\delta \rightarrow 0} \frac{\log N_{\delta}(M)}{-\log \delta} \text { and } \overline{\operatorname{dim}_{B}}(M):=\limsup _{\delta \rightarrow 0} \frac{\log N_{\delta}(M)}{-\log \delta} .
$$

When these numbers are equal, the common value will be the box dimension of $M$, that is,

$$
\operatorname{dim}_{B}(M):=\lim _{\delta \rightarrow 0} \frac{\log N_{\delta}(M)}{-\log \delta} .
$$

For more details on the above definitions and their properties, see $[13$, Chapters 2-3]. Notice that, in particular,

$$
\operatorname{dim}_{H}(M) \leq \underline{\operatorname{dim}_{B}}(M) \leq \overline{\operatorname{dim}_{B}}(M)
$$

for any subset $M$ of $\mathbb{R}^{n}$. 
As usual, we endow the space $\mathcal{C}[0,1]$ of continuous functions $[0,1] \rightarrow \mathbb{R}$ with the maximum norm. Observe that it is an infinite dimensional separable Banach space, hence its dimension is exactly $\mathfrak{c}$, where the last symbol stands for the continuum (as usual).

In this paper, we are interested in the following type of question:

Consider the subset $\mathcal{H}$ of continuous functions $f:[0,1] \rightarrow \mathbb{R}$ with $\operatorname{dim}_{H}(g r a p h(f))=s$. Is there an infinite dimensional subspace $X \subset \mathcal{H} \cup\{0\}$ every non-zero element of which has Hausdorff dimension $s$ ? What about the same problem replacing Hausdorff dimension by Box dimension?

This kind of questions (related ones are dealt with in $[1,9]$ ) fall under the area of research nowadays known as lineability and spaceability. Let us provide a brief summary of the definitions from this area and that we shall need along this paper.

Assume that $X$ is a vector space and $\alpha$ is a cardinal number. Then a subset $A \subset X$ is said to be:

- lineable if there is an infinite dimensional vector space $M$ such that $M \backslash\{0\} \subset A$.

- $\alpha$-lineable if there exists a vector space $M$ with $\operatorname{dim}(M)=\alpha$ and $M \backslash\{0\} \subset A$.

- maximal-lineable if it is $\operatorname{dim}(X)$-lineable.

If, in addition, $X$ is a topological vector space, then the subset $A$ is said to be:

- spaceable in $X$ whenever there is a closed infinite-dimensional vector subspace $M$ of $X$ such that $M \backslash\{0\} \subset A$.

- dense-lineable in $X$ whenever there is a dense vector subspace $M$ of $X$ satisfying $M \backslash\{0\} \subset A$.

- $\alpha$-dense-lineable in $X$ whenever there is a dense vector subspace $M$ of $X$ with $\operatorname{dim}(M)=\alpha$ and $M \backslash\{0\} \subset A$.

- maximal-dense-lineable in $X$ whenever there is a dense vector subspace $M$ of $X$ with $\operatorname{dim}(M)=\operatorname{dim}(X)$ and $M \backslash\{0\} \subset A$.

And, provided that $X$ is a vector space contained in some (linear) algebra, then $A$ is called:

- algebrable if there is an algebra $M$ so that $M \backslash\{0\} \subset A$ and $M$ is infinitely generated, that is, the cardinality of any system of generators of $M$ is infinite.

- strongly $\alpha$-algebrable if there exists an $\alpha$-generated free algebra $M$ with $M \backslash\{0\} \subset A$. Recall that if $X$ is contained in a commutative algebra, then a set $B \subset X$ is a generating set of some free algebra contained in $A$ if and only if for any $N \in \mathbb{N}$, any nonzero polynomial $P$ in $N$ variables without constant term and any distinct $f_{1}, \ldots, f_{N} \in B$, we have $P\left(f_{1}, \ldots, f_{N}\right) \in A \backslash\{0\}$. 
For an account of results on lineability, the reader is referred to the survey [10], the monograph [2], and the works $[4,11,12,14,20]$ in order to see the many fields in which this theory has made an impact so far.

Here, $I$ shall denote a non-trivial interval of $\mathbb{R}$. The following definition and proposition can be found in [6] or [13].

Definition 1.1. Let $f: I \rightarrow \mathbb{R}$.

(1) We say that $f$ is Hölder continuous with exponent $\alpha>0$ if there exist $c, \delta>0$ such that

$$
|f(x)-f(y)| \leq c|x-y|^{\alpha}
$$

for every $x, y \in I$ such that $|x-y|<\delta$.

(2) We say that $f$ satisfies the lower Hölder condition, with exponent $\beta>0$, if there exist $\tilde{\delta}, \tilde{c}>0$ such that

$$
\sup _{x \in J} f(x)-\inf _{x \in J} f(x) \geq \tilde{c}|J|^{\beta}
$$

for every interval $J \subset I$ with $|J|<\tilde{\delta}$.

Proposition 1.2. If $f: I \rightarrow \mathbb{R}$ is Hölder continuous with exponent $\alpha \in$ $(0,1]$, then

$$
\overline{\operatorname{dim}_{B}}(\operatorname{graph}(f)) \leq 2-\alpha .
$$

If a continuous function $f: I \rightarrow \mathbb{R}$ satisfies the lower Hölder continuous condition with exponent $\beta \in(0,1]$, then

$$
\underline{\operatorname{dim}_{B}}(\operatorname{graph}(f)) \geq 2-\beta .
$$

This paper is arranged in two main sections. Section 2 shall deal with continuous functions with prescribed Box and Hausdorff dimension of the graph and the problem of lineability of this class of functions. Section 3 will cover the same problem but, in this case, in relation to the Box dimension everywhere. Open questions and directions of research shall also be provided within this topic.

\section{On CONTINuOUS Functions With PRESCRIBED Box AND HAUSDORFF DIMENSION OF THEIR GRAPHS}

Let us, first, recall a series of results that will be useful for the purpose of this paper. The first one is a direct consequence of the basic properties of the Hausdorff measure.

Lemma 2.1. The Hausdorff dimension of a subset of $\mathbb{R}^{2}$ is invariant under translations and dilations.

Lemma 2.2. Given a number $s \in[1,2]$, a compact interval $[a, b]$ and two real numbers $v, w$, there exists a continuous function $f:[a, b] \rightarrow \mathbb{R}$ such that $f(a)=v, f(b)=w$ and $\operatorname{dim}_{H}(\operatorname{graph}(f))=s$. 
Proof. The case $s=1$ is straightforward. We know that the Hausdorff dimension of the graph of the Weierstrass function on $[0,1]$ is $D=2+\frac{\log \lambda}{\log b}$. Therefore, given $s \in(1,2)$, the functions $F_{s}:=W_{\lambda, b}$ have a graph with Hausdorff dimension $s$ for a suitable choice of $\lambda$ and $b$. If $s=2$, then the function

$$
F_{2}(x)=\sum_{n=1}^{\infty} n^{-\sqrt{n}} \sin \left(n^{n} 2 \pi x\right)
$$

has graph of Hausdorff dimension 2 (see [5] and Figure 2).

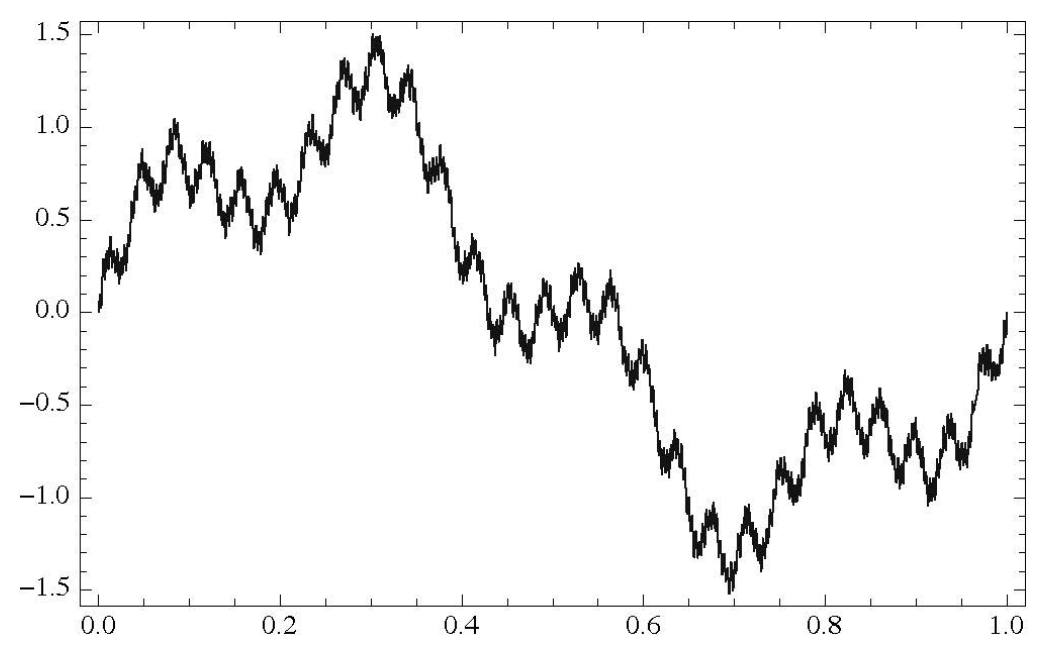

Figure 2. A sketch of function $F_{2}$ from the proof of Lemma 2.2. Here, $\operatorname{graph}\left(F_{2}\right)$ has Hausdorff dimension 2.

By Lemma 2.1, we can translate and dilate, for any $s \in(1,2]$, the function $F_{s}$ in order to obtain $\tilde{F}_{s}:[a, b] \rightarrow \mathbb{R}$ with $\operatorname{dim}_{H}\left(\operatorname{graph}\left(\tilde{F}_{s}\right)\right)=s$.

Finally take into account that, according to [18, Theorem 1], the Hausdorff dimension of the graph of a function does not change by adding a Lipschitz function, so that we can add a suitable affine function in order to fulfill the property on the extremes of the interval.

Theorem 2.3. Given $s \in(1,2]$, the set of continuous functions $f:[0,1] \rightarrow$ $\mathbb{R}$ with $\operatorname{dim}_{H}($ graph $(f))=s$ is spaceable.

Proof. Fixed $s \in(1,2]$ we split $[0,1]$ into infinitely many intervals with disjoint interior as follows:

$$
[0,1]=\{1\} \cup \bigcup_{n \in \mathbb{N}} I_{n},
$$

where $I_{n}=\left[1-\frac{1}{n}, 1-\frac{1}{n+1}\right]$ for $n \in \mathbb{N}$.

Now, by Lemma 2.2 , for every $n \in \mathbb{N}$ there exists a continuous function $f_{n}:[0,1] \rightarrow \mathbb{R}$ such that: 
(i) $f_{n}\left(1-\frac{1}{n}\right)=0$,

(ii) $f_{n}\left(1-\frac{1}{n+1}\right)=0$,

(iii) $f_{n}$ is null on $[0,1] \backslash I_{n}$,

(iv) $\left\|f_{n}\right\|_{\infty}<1 / n$, and

(v) $\operatorname{dim}_{H}\left(\operatorname{graph}\left(f_{n}\right)\right)=s$.

It is clear that the family $\left\{f_{n}: n \in \mathbb{N}\right\}$ is linearly independent (since the interior of their corresponding supports are pairwise disjoint). Next, let $M$ be the closed linear span of $\left\{f_{n}: n \in \mathbb{N}\right\}$. Again due the disjointness of the interior of the supports, every nonzero member of $M$ is a finite or infinite sum of $f_{n}$ 's that, on each $I_{n}$, is a multiple of the corresponding $f_{n}$. Therefore its graph has Hausdorff dimension $s$. By (i) and (ii), any nonzero member of $M$ is obviously continuous on $[0,1$ ) (observe that, possibly, the supports of the functions $f_{n}$ 's included in the member of $M$ may accumulate at 1 ). Finally, condition (iv) and the fact that $f_{n}(1)=0$, guarantee the continuity at 1 .

Lemma 2.4. Let $s \in(1,2]$, the set of functions $f \in \mathcal{C}[0,1]$ with

$$
\operatorname{dim}_{H}\left(\operatorname{graph}_{[0,1]}(f)\right)=\operatorname{dim}_{B}\left(\operatorname{graph}_{[0,1]}(f)\right)=s .
$$

is $\mathfrak{c}$-lineable.

Proof. Let $s \in(1,2)$ and $M=\operatorname{span}\left\{f_{a}: 0 \leq a<1\right\}$ where

$$
f_{a}(x)=\left\{\begin{array}{cc}
W_{\lambda, b}\left(\frac{x-a}{1-a}\right)-W_{\lambda, b}(0) & \text { if } x \in[a, 1], \\
0 & \text { if } x<a,
\end{array}\right.
$$

with parameters $b \geq 2$ and $1 / b<\lambda<1$ chosen such that

$$
\operatorname{dim}_{H}\left(\operatorname{graph}_{[0,1]}\left(f_{a}\right)\right)=s .
$$

To show the linear independence, we shall proceed by induction. First, we show that for any two functions $\left\{f_{a_{1}}, f_{a_{2}}\right\}$ are linearly independent with $a_{1}<a_{2}$. Suppose that $\lambda_{1} f_{a_{1}}+\lambda_{2} f_{a_{2}}=0$. Then for any $x \in\left(a_{1}, a_{2}\right)$, $\lambda_{1} f_{a_{1}}(x)+\lambda_{2} f_{a_{2}}(x)=\lambda_{1} f_{a_{1}}(x)=0$, thus $\lambda_{1}=0$ and hence $\lambda_{2}=0$.

Now suppose that any set $\left\{f_{a_{i}}: 0 \leq a_{1}<\cdots a_{l}<1\right\}$ with $l \leq N-1$ is linearly independent. Let us suppose that $\lambda_{1} f_{a_{1}}+\cdots+\lambda_{N} f_{a_{N}}=0$, where $0 \leq a_{1}<a_{2}<\cdots a_{N}<1$. Thus, for any $x \in\left(a_{1}, a_{2}\right)$

$$
\lambda_{1} f_{a_{1}}(x)+\cdots+\lambda_{N} f_{a_{N}}(x)=\lambda_{1} f_{a_{1}}(x)=0 .
$$

Thus, $\lambda_{1}=0$ and by inductional assumption, hence $\lambda_{2}=\cdots=\lambda_{N}=0$.

Now, we will show that for any non-trivial combination $\left(\lambda_{i} \neq 0\right.$ for $1 \leq i \leq N)$ with $0 \leq a_{1}<a_{2}<\cdots a_{N}<1$

$$
\begin{gathered}
\operatorname{dim}_{H}\left(\operatorname{graph}_{[0,1]}\left(\lambda_{1} f_{a_{1}}+\cdots+\lambda_{N} f_{a_{N}}\right)\right) \\
=\operatorname{dim}_{B}\left(\operatorname{graph}_{[0,1]}\left(\lambda_{1} f_{a_{1}}+\cdots+\lambda_{N} f_{a_{N}}\right)\right)=s .
\end{gathered}
$$


The upper bound of $\overline{\operatorname{dim}}_{B}\left(\operatorname{graph}_{[0,1]}\left(\lambda_{1} f_{a_{1}}+\cdots+\lambda_{N} f_{a_{N}}\right)\right) \leq s$ by the Hölder continuity of $\lambda_{1} f_{a_{1}}+\cdots+\lambda_{N} f_{a_{N}}$. On the other hand,

$$
\begin{aligned}
& \operatorname{dim}_{H}\left(\operatorname{graph}_{[0,1]}\left(\lambda_{1} f_{a_{1}}+\cdots+\lambda_{N} f_{a_{N}}\right) \geq\right. \\
& \geq \operatorname{dim}_{H}\left(\operatorname{graph}_{\left[a_{1}, a_{2}\right]}\left(\lambda_{1} f_{a_{1}}+\cdots+\lambda_{N} f_{a_{N}}\right)\right)= \\
& =\operatorname{dim}_{H}\left(\operatorname{graph}_{\left[a_{1}, a_{2}\right]}\left(\lambda_{1} f_{a_{1}}\right)\right)= \\
& =\operatorname{dim}_{H}\left(\operatorname{graph}_{\left[a_{1}, a_{2}\right]}\left(f_{a_{1}}\right)\right)=s .
\end{aligned}
$$

Now, since $\operatorname{dim}_{H}(M) \leq \operatorname{dim}_{B}(M) \leq \overline{\operatorname{dim}_{B}}(M)$, we obtain the result.

For $s=2$, we change $\overline{W_{\lambda, b}}$ by

$$
F_{2}(x)=\sum_{n=1}^{\infty} n^{-\sqrt{n}} \sin \left(n^{n} 2 \pi x\right) .
$$

We shall now need an auxiliary, general result about lineability: Let $X$ be a vector space and $A, B$ be two subsets of $X$. According to [3], we say that $A$ is stronger than $B$ whenever $A+B \subset A$. The following lemma (of which many variants have been proved) can be found in $[2,3,10]$ and references therein.

Lemma 2.5. Assume that $X$ is a metrizable topological vector space. Let $A \subset X$ be (maximal) lineable. Suppose that there exists a dense-lineable subset $B \subset X$ such that $A$ is stronger than $B$ and $A \cap B=\varnothing$. Then $A$ is (maximal) dense-lineable in $X$.

Theorem 2.6. Given $s \in(1,2]$, the set of functions $f \in \mathcal{C}[0,1]$ with

$$
\operatorname{dim}_{H}(\operatorname{graph}(f))=\operatorname{dim}_{B}(\operatorname{graph}(f))=s
$$

is maximal dense-lineable in $\mathcal{C}[0,1]$.

Proof. Denote

$$
A(s):=\left\{f \in \mathcal{C}[0,1]: \operatorname{dim}_{H}(\operatorname{graph}(f))=\operatorname{dim}_{B}(\operatorname{graph}(f))=s\right\} .
$$

It is straightforward that the set $P$ of all polygonal lines (i.e., continuous piecewise affine functions) is a dense linear subset of $\mathcal{C}[0,1]$. In addition, we have that for any $s \in(1,2], A(s)$ is stronger than $P$ because

$$
\operatorname{dim}_{H}(\operatorname{graph}(f+h))=\operatorname{dim}_{H}(\operatorname{graph}(f))
$$

and

$$
\operatorname{dim}_{B}(\operatorname{graph}(f+h))=\operatorname{dim}_{B}(\operatorname{graph}(f))
$$

for every Lipschitz functions $h$ (see, [18, Theorem 1], [5]).

Finally, taking into account that $A(s)$ is c-lineable by Lemma 2.4 , a direct application of Lemma 2.5 helps us to conclude the proof. 
All previous results can be extended to higher dimensions just by using properties regarding the Hausdorff dimension and cartesian product of sets.

It is well known that given any pair of subsets $E \subset \mathbb{R}^{n}$ and $F \subset \mathbb{R}^{m}$, we only can assert that

$$
\operatorname{dim}_{H}(E \times F) \geq \operatorname{dim}_{H}(E)+\operatorname{dim}_{H}(F) .
$$

But if one of the subsets is regular enough, the equality holds (see [13, Section 7.1]).

Lemma 2.7. For any pair of subsets $E \subset \mathbb{R}^{n}$ and $F \subset \mathbb{R}^{m}$ with $\operatorname{dim}_{H}(F)=$ $\overline{\operatorname{dim}_{B}}(F)$, then

$$
\operatorname{dim}_{H}(E \times F)=\operatorname{dim}_{H}(E)+\operatorname{dim}_{H}(F) .
$$

This result is false in general, if we change Hausdorff-dimension by any type of Box-dimension.

Theorem 2.8. Given $N \in \mathbb{N}$ and $S \in(N, N+1]$, the set of functions $F:[0,1]^{N} \rightarrow \mathbb{R}$ such that its graph, $\operatorname{graph}(F)=\left\{\left(x_{1}, \ldots, x_{N}, F\left(x_{1}, \ldots, x_{N}\right)\right) \in \mathbb{R}^{N+1}, x_{i} \in[0,1], 1 \leq i \leq N\right\}$, has Hausdorff dimension exactly $S$, is spaceable and maximal dense-lineable in $\mathcal{C}\left([0,1]^{N}\right)$.

Proof. Fix a $f \in \mathcal{C}([0,1])$ and define $\tilde{f}:[0,1]^{N} \rightarrow \mathbb{R}$ as $\tilde{f}\left(x_{1}, \cdots, x_{N}\right)=$ $f\left(x_{N}\right)$. This function is clearly continuous on $[0,1]^{N}$, in addition we have that $\operatorname{graph}(\tilde{f})=[0,1]^{N-1} \times \operatorname{graph}(f)$ and by applying Lemma 2.7 with $F=[0,1]^{N-1}$, it holds that

$$
\operatorname{dim}_{H}(\operatorname{graph}(\tilde{f}))=N-1+\operatorname{dim}_{H}(\operatorname{graph}(f)) .
$$

Now fix $S \in(N, N+1]$ and let $M$ be the infinite-dimensional closed vector space of $\mathcal{C}([0,1])$ given by Theorem 2.3 for $s=S-N+1 \in(1,2]$. It is straightforward that the set $\widetilde{M}:=\{\tilde{f}: f \in M\}$ is an infinite dimensional closed vector space of $\mathcal{C}\left([0,1]^{N}\right)$ all of whose nonzero functions have graph with Hausdorff dimension $N-1+s=S$.

A similar construction, based on Theorem 2.6, gives the maximal-dense lineability.

By the other hand, one may think that the same arguments as those used in Theorem 2.3 would imply that the set of continuous functions on $[0,1]$ with prescribed Box dimension $s \in(1,2]$ is spaceable. This is not true because, as it is shown in [13, Example 3.5], the Box dimension is not stable under numerable (disjoint) unions.

In [5, Corollary 8] Baranski construct for every $h, b \in[1,2]$ with $h \leq b$ a function $f \in \mathcal{C}([0,1])$ such that

$$
\operatorname{dim}_{H}(\operatorname{graph}(f))=\underline{\operatorname{dim}_{B}}(\operatorname{graph}(f))=h \text { and } \overline{\operatorname{dim}_{B}}(\operatorname{graph}(f))=b .
$$

As a matter of fact, using this function, the stability of the Box dimension under finite unions and Lemma 2.5, the following result can also be proved. 
Corollary 2.9. Given $h, b \in[1,2]$ with $h \leq b$, the set of functions $f \in \mathcal{C}[0,1]$ with

$$
\operatorname{dim}_{H}(\operatorname{graph}(f))=\underline{\operatorname{dim}_{B}}(\operatorname{graph}(f))=h \quad \text { and } \overline{\operatorname{dim}_{B}}(\operatorname{graph}(f))=b
$$

is dense-lineable in $\mathcal{C}[0,1]$.

In order to motivate the next question, we prove the next result.

Proposition 2.10. Let $g \in C^{1}(\mathbb{R})$ be non-constant function such that $R_{g}=$ $\left\{x \in \mathbb{R}: g^{\prime}(x)=0\right\}$ is finite. Then for any continuous function $f:[0,1] \rightarrow \mathbb{R}$

$$
\operatorname{dim}_{H}\left(\operatorname{graph}_{[0,1]}(g \circ f)\right)=\operatorname{dim}_{H}\left(\operatorname{graph}_{[0,1]}(f)\right) .
$$

In particular, $\operatorname{dim}_{H}\left(\operatorname{graph}_{[0,1]}\left(f^{n}\right)\right)=\operatorname{dim}_{H}\left(\operatorname{graph}_{[0,1]}(f)\right)$ for all $n$.

Proof. First, we introduce the notation of convex hull. Namely, for a Borel set $A \subset[0,1]$ let $\operatorname{conv}(A)$ the smallest closed interval such that $A \subset \operatorname{conv}(A)$.

Observe that for any Borel set $A \subset[0,1]$ and any bi-Lipschitz map $g$ : $\operatorname{conv}(f(A)) \rightarrow g(\operatorname{conv}(f(A)))$

$$
\operatorname{dim}_{H} \operatorname{graph}_{A}(g \circ f)=\operatorname{dim}_{H} \operatorname{graph}_{A}(f) .
$$

Indeed, $G:(x, y) \rightarrow(x, g(y))$ is also a bi-Lipschiz map on $\operatorname{conv}(A) \times$ $\operatorname{conv}(f(A))$, moreover $\operatorname{graph}_{A}(g \circ f)=G\left(\operatorname{graph}_{A}(f)\right)$. Equation (2.1) follows by the bi-Lipschitz invariance of the Hausdorff dimension.

Let $g \in C^{1}(\mathbb{R})$ be non-constant function such that $R_{g}=\left\{x \in \mathbb{R}: g^{\prime}(x)=\right.$ $0\}$ is finite. Then there exists $N$ such that for every $n \geq N, f([0,1] \backslash$ $\cup_{y \in R_{g}}(y-1 / n, y+1 / n)$ is a finite union of disjoint closed intervals. Denote $I_{1}^{n}, \cdots, I_{m}^{n}$ this collection of disjoint closed intervals. It is easy to see that $g: I_{l}^{n} \rightarrow g\left(I_{l}^{n}\right)$ is a bi-Lipschitz map. Moreover,

$$
[0,1]=\left\{x: f(x) \in R_{g}\right\} \cup \bigcup_{n=N}^{\infty} \bigcup_{l=1}^{m} f^{-1}\left(I_{l}^{n}\right) .
$$

by (2.1), for every $n \geq N$ and $1 \leq l \leq m$

$$
\operatorname{dim}_{H}\left(\operatorname{graph}_{f^{-1}\left(I_{l}^{n}\right)}(g \circ f)\right)=\operatorname{dim}_{H}\left(\operatorname{graph}_{f^{-1}\left(I_{l}^{n}\right)}(f)\right) .
$$

Hence, by the countable stability of the Hausdorff dimension

$$
\begin{aligned}
& \operatorname{dim}_{H}\left(\operatorname{graph}_{[0,1]}(g \circ f)\right) \\
& =\operatorname{dim}_{H}\left(\operatorname{graph}_{\left\{x: f(x) \in R_{g}\right\}}(g \circ f) \cup \bigcup_{n=N}^{\infty} \bigcup_{l=1}^{m} \operatorname{graph}_{f^{-1}\left(I_{l}^{n}\right)}(g \circ f)\right)
\end{aligned}
$$

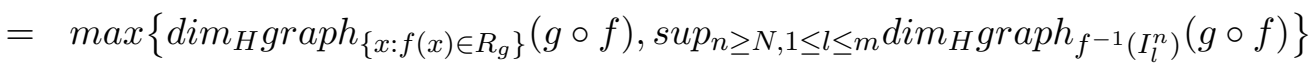

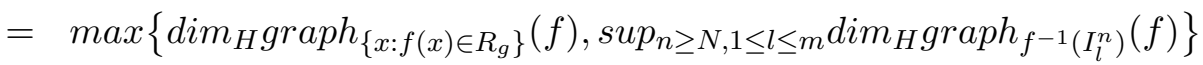

$$
\begin{aligned}
& =\operatorname{dim}_{H}\left(\operatorname{graph}_{\left\{x: f(x) \in R_{g}\right\}}(f) \cup \bigcup_{n=N}^{\infty} \bigcup_{l=1}^{m} \operatorname{graph}_{f^{-1}\left(I_{l}^{n}\right)}(f)\right) \\
& =\operatorname{dim}_{H}\left(\operatorname{graph}_{[0,1]}(f)\right) \text {. }
\end{aligned}
$$


Note that we also used that the set $R_{g}$ is finite and thus,

$$
\operatorname{dim}_{H}\left(\operatorname{graph}_{\left\{x: f(x) \in R_{g}\right\}}(g \circ f)\right), \operatorname{dim}_{H}\left(\operatorname{graph}_{\left\{x: f(x) \in R_{g}\right\}}(f)\right) \leq 1 .
$$

We finish this section posing an open question. We propose it in the one-dimensional case, but it is easily adapted to higher dimensions.

Question 2.11. Given $s \in(1,2]$, it is possible to obtain the algebrability of the set of functions $f \in \mathcal{C}[0,1]$ with $\operatorname{dim}_{H}(\operatorname{graph}(f))=s$ ?

\section{On CONTINUOUS FUnCTIONS With PRESCRIBED BOX DiMENSION EVERYWHERE OF THEIR GRAPHS}

Let us consider the following definition, that shall be recurrent from now on.

Definition 3.1. We say that a function has Box dimension $\alpha$ everywhere in $[0,1]$ (or, simply, everywhere) if

$$
\operatorname{dim}_{B}\left(\operatorname{graph}_{(a, b)}(f)\right)=\alpha \quad \forall(a, b) \subset[0,1],
$$

that is, the graph of $f$ has Box dimension $\alpha$ if restricted to any (nonvoid) open subinterval of $[0,1]$.

It is known (see [13, Example 11.3]) that the classical Weierstrass functions have Box dimension $D=2+\frac{\log \lambda}{\log b}$ everywhere on $[0,1]$.

Invoking Hölder continuity, we can establish also the maximal denselineability of continuous functions having graph with (different) lower and upper box dimensions everywhere between any two numbers $0<s_{1}<s_{2}<$ 1.

Theorem 3.2. Given $0<\alpha<\beta<1$, the set of functions $f:[0,1] \rightarrow$ $\mathbb{R}$ Hölder continuous with exponent $\alpha$ and lower Hölder continuous with exponent $\beta$ is $\mathfrak{c}$-lineable.

Proof. Given $0<\alpha<\beta<1$, we define for a sufficiently large prime number $p$ (that shall be specified later in the proof) the following:

$$
V_{\alpha, \beta}:=\operatorname{span}\left\{f_{\gamma}(t):=\sum_{n=0}^{\infty} p^{-\gamma n} \sin \left(2 \pi p^{n} t\right): \alpha<\gamma<\beta\right\} .
$$

Then $V_{\alpha, \beta}$ is a linear subspace of Hölder continuous functions with exponent $\alpha$, since each $f_{\gamma}$ is Hölder continuous with exponent $\gamma$. Moreover the set

$$
\left\{f_{\gamma}: \alpha<\gamma<\beta\right\}
$$

is linearly independent. Indeed, let us suppose that

$$
\lambda_{1} f_{\gamma_{1}}+\lambda_{2} f_{\gamma_{2}}+\cdots+\lambda_{k} f_{\gamma_{k}}=0
$$


for some $k \in \mathbb{N}, \alpha<\gamma_{i}<\beta$ for every $i \in\{1,2, \ldots, k\}$ and $\lambda_{1}, \ldots, \lambda_{k} \in \mathbb{R}$.

Then

$$
\sum_{n=0}^{\infty}\left(\lambda_{1} p^{-\gamma_{1} n}+\lambda_{2} p^{-\gamma_{2} n}+\cdots+\lambda_{k} p^{-\gamma_{k} n}\right) \sin \left(2 \pi p^{n} t\right)=0
$$

for every $t \in[0,1]$, so the uniqueness of coefficients of the Fourier series yields

$$
\lambda_{1} p^{-\gamma_{1} n}+\lambda_{2} p^{-\gamma_{2} n}+\cdots+\lambda_{k} p^{-\gamma_{k} n}=0
$$

for every $n \in \mathbb{N}_{0}$. For $n=0, \ldots, k-1$, we obtain the following conditions:

$$
\left\{\begin{array}{c}
\lambda_{1}+\lambda_{2}+\cdots+\lambda_{k}=0 \\
\lambda_{1} p^{-\gamma_{1}}+\lambda_{2} p^{-\gamma_{2}}+\cdots+\lambda_{k} p^{-\gamma_{k}}=0 \\
\vdots \\
\lambda_{1} p^{-\gamma_{1}(k-1)}+\lambda_{2} p^{-\gamma_{2}(k-1)}+\cdots+\lambda_{k} p^{-\gamma_{k}(k-1)}=0 .
\end{array}\right.
$$

The above equations are equivalent to the following one.

$$
\left(\begin{array}{cccc}
1 & 1 & \cdots & 1 \\
p^{-\gamma_{1}} & p^{-\gamma_{2}} & \cdots & p^{-\gamma_{k}} \\
\vdots & \vdots & \ddots & \vdots \\
p^{-\gamma_{1}(k-1)} & p^{-\gamma_{2}(k-1)} & \cdots & p^{-\gamma_{k}(k-1)}
\end{array}\right)\left(\begin{array}{c}
\lambda_{1} \\
\lambda_{2} \\
\vdots \\
\lambda_{k}
\end{array}\right)=\left(\begin{array}{c}
0 \\
0 \\
\vdots \\
0
\end{array}\right) .
$$

The previous square matrix is a Vandermonde-type matrix and, thus, its determinant is not 0 since $p^{-\gamma_{1}}, \ldots, p^{-\gamma_{k}}$ are different and non-zero. Therefore, $\lambda_{1}=0, \lambda_{2}=0, \ldots, \lambda_{k}=0$. Thus $\operatorname{dim} V_{\alpha, \beta}=\mathfrak{c}$.

Now, we shall prove that every function $f \in V_{\alpha, \beta} \backslash\{0\}$ is lower Hölder continuous with exponent $\beta$.

If $f \in V_{\alpha, \beta} \backslash\{0\}$, then there are $N \in \mathbb{N}, \alpha<\gamma_{1}<\gamma_{2}<\cdots<\gamma_{N}<\beta$ and $\lambda_{1}, \ldots, \lambda_{N} \in \mathbb{R} \backslash\{0\}$ such that

$$
f=\lambda_{1} f_{\gamma_{1}}+\cdots+\lambda_{N} f_{\gamma_{N}} .
$$

We can assume that $\lambda_{1}=1$. If that were not the case, then we would prove the property of lower Hölder condition with exponent $\beta$ for the function

$$
g=f_{\gamma_{1}}+\frac{\lambda_{2}}{\lambda_{1}} f_{\gamma_{2}}+\cdots+\frac{\lambda_{N}}{\lambda_{1}} f_{\gamma_{N}}
$$

and then $f=\lambda_{1} g$ would also satisfy the lower Hölder condition with exponent $\beta$.

For a sufficiently large positive integer $n$ (to be specified later in the proof), setting $\delta=p^{-n}$, we may choose a subinterval $\left[i \delta,\left(i+\frac{1}{8}\right) \delta\right]$ in each 
interval $[i \delta,(i+1) \delta],(i \in \mathbb{N})$, and denote these intervals by $\left[s_{i}, t_{i}\right]$. By doing this, we would have that

$$
\begin{aligned}
\left|f_{\gamma_{1}}\left(s_{i}\right)-f_{\gamma_{1}}\left(t_{i}\right)\right| \geq & \mid p^{-\gamma_{1} n}\left(\sin \left(p^{n} 2 \pi s_{i}\right)-\sin \left(p^{n} 2 \pi t_{i}\right) \mid\right. \\
& -\mid \sum_{k=1}^{n-1} p^{-\gamma_{1} k}\left(\sin \left(p^{k} 2 \pi s_{i}\right)-\sin \left(p^{k} 2 \pi t_{i}\right) \mid\right. \\
& -\mid \sum_{k=n+1}^{\infty} p^{-\gamma_{1} k}\left(\sin \left(p^{k} 2 \pi s_{i}\right)-\sin \left(p^{k} 2 \pi t_{i}\right) \mid\right. \\
\geq & 2 \pi p^{\left(1-\gamma_{1}\right) n}\left|\cos \left(p^{n} 2 \pi s_{i}^{\prime}\right)\right|\left|t_{i}-s_{i}\right| \\
& -2 \pi\left|t_{i}-s_{i}\right| \sum_{k=0}^{n-1} p^{\left(1-\gamma_{1}\right) k}-2 \sum_{k=n+1}^{\infty} p^{-\gamma_{1} k} \\
\geq & \frac{\sqrt{2} \pi}{8} p^{-\gamma_{1} n}-\frac{\pi}{4} p^{-n} \sum_{k=0}^{n-1} p^{\left(1-\gamma_{1}\right) k}-2 \sum_{k=n+1}^{\infty} p^{-\gamma_{1} k} \\
\geq & \frac{\sqrt{2} \pi}{8} p^{-\gamma_{1} n}-\frac{\pi}{4} \frac{p^{\gamma_{1}-1}}{1-p^{\gamma_{1}-1}} p^{-\gamma_{1} n}-2 \frac{p^{-\gamma_{1}}}{1-p^{-\gamma_{1}}} p^{-\gamma_{1} n} \\
\geq & C_{1} p^{-\gamma_{1} n},
\end{aligned}
$$

with $C_{1}>0$ for a suitable choice of large values of $p$, as we advanced earlier in this proof. Moreover,

$$
\begin{aligned}
\left|f_{\gamma_{j}}\left(s_{i}\right)-f_{\gamma_{j}}\left(t_{i}\right)\right| \leq & \mid \sum_{k=1}^{n} p^{-\gamma_{j} k}\left(\sin \left(p^{k} 2 \pi s_{i}\right)-\sin \left(p^{k} 2 \pi t_{i}\right) \mid\right. \\
& +\mid \sum_{k=n+1}^{\infty} p^{-\gamma_{j} k}\left(\sin \left(p^{k} 2 \pi s_{i}\right)-\sin \left(p^{k} 2 \pi t_{i}\right) \mid\right. \\
\leq & 2 \pi \sum_{k=1}^{n} p^{\left(1-\gamma_{j}\right) k}\left|\cos \left(p^{k} 2 \pi s_{i}^{\prime}\right)\right|\left|t_{i}-s_{i}\right|+2 \sum_{k=n+1}^{\infty} p^{-\gamma_{j} k} \\
\leq & \frac{2 \pi \delta}{8} \sum_{k=1}^{n} p^{\left(1-\gamma_{j}\right) k}+2 \sum_{k=n+1}^{\infty} p^{-\gamma_{j} k} \\
\leq & \frac{\pi}{4} p^{-n} \sum_{k=1}^{n} p^{\left(1-\gamma_{j}\right) k}+2 \sum_{k=n+1}^{\infty} p^{-\gamma_{j} k} \\
\leq & \frac{\pi}{4} \frac{p^{1-\gamma_{j}}}{p^{1-\gamma_{j}}-1} p^{-\gamma_{j} n}+2 \frac{p^{-\gamma_{j}}}{1-p^{-\gamma_{j}}} p^{-\gamma_{j} n} \\
\leq & C_{i} p^{-\gamma_{j} n},
\end{aligned}
$$


for certain $C_{i}>0$. Thus, for every $i$,

$$
\begin{aligned}
\left|f\left(s_{i}\right)-f\left(t_{i}\right)\right| \geq & \left|f_{\gamma_{1}}\left(s_{i}\right)-f_{\gamma_{1}}\left(t_{i}\right)\right|-\left|\lambda_{2}\right|\left|f_{\gamma_{2}}\left(s_{i}\right)-f_{\gamma_{2}}\left(t_{i}\right)\right|-\ldots \\
& -\left|\lambda_{N}\right|\left|f_{\gamma_{N}}\left(s_{i}\right)-f_{\gamma_{N}}\left(t_{i}\right)\right| \\
\geq & C_{1} p^{-\gamma_{1} n}-\left|\lambda_{2}\right| C_{2} p^{-\gamma_{2} n}-\ldots-\left|\lambda_{N}\right| C_{N} p^{-\gamma_{N} n} \\
\geq & C_{1} p^{-\gamma_{1} n}-\left|\lambda_{2}\right| C_{2} p^{-\gamma_{2} n}-\ldots-\left|\lambda_{N}\right| C_{N} p^{-\gamma_{N} n} \\
= & \left(C_{1}-\left|\lambda_{2}\right| C_{2} p^{\left(\gamma_{1}-\gamma_{2}\right) n}-\ldots-\left|\lambda_{N}\right| C_{N} p^{\left(\gamma_{1}-\gamma_{N}\right) n}\right) p^{-\gamma_{1} n} \\
\geq & C p^{-\beta n}
\end{aligned}
$$

with $C>0$ provided we have chosen large values of $n$ (as we mentioned earlier in this proof). This finishes the proof.

Theorem 3.3. Given $1<s_{1}<s_{2}<2$, the set of functions $f \in \mathcal{C}[0,1]$ with

$$
s_{1}<\underline{\operatorname{dim}_{B}}(\operatorname{graph}(f))<\overline{\operatorname{dim}_{B}}(\operatorname{graph}(f))<s_{2}
$$

everywhere in $[0,1]$ is maximal dense-lineable in $\mathcal{C}([0,1])$.

Proof. Let $B\left(s_{1}, s_{2}\right)$ denote the set of functions $f \in \mathcal{C}[0,1]$ with

$$
s_{1}<\underline{\operatorname{dim}_{B}}(\operatorname{graph}(f))<\overline{\operatorname{dim}_{B}}(\operatorname{graph}(f))<s_{2}
$$

everywhere in $[0,1]$. The set $P$ of all polygonal lines is a dense linear subset of $\mathcal{C}[0,1]$. In addition, by [5], we have that $B\left(s_{1}, s_{2}\right)$ is stronger than $P$. Finally, taking into account that $B\left(s_{1}, s_{2}\right)$ is maximal lineable as consequence of Theorem 3.2 for $\alpha>2-s_{2}, \beta<2-s_{1}$, a direct application of Lemma 2.5 concludes the proof.

We finish this section with the following open question.

Question 3.4. Given $s \in(1,2)$, is it possible to obtain maximal-denselineability (or algebrability) of the set of functions $f \in \mathcal{C}[0,1]$ whose graph have Box dimension s everywhere in $[0,1]$ ?

Acknowledgments. The authors would like to thank the anonymous referee for his/her suggestions and comments (specially regarding Lemma 2.4 and Proposition 2.10) which helped improving the results in the paper.

\section{REFERENCES}

[1] N. Albuquerque, L. Bernal-González, D. Pellegrino, and J. B. Seoane-Sepúlveda, Peano curves on topological vector spaces, Linear Algebra Appl. 460 (2014), 81-96, DOI 10.1016/j.laa.2014.07.029.

[2] R. M. Aron, L. Bernal González, D. M. Pellegrino, and J. B. Seoane Sepúlveda, Lineability: the search for linearity in mathematics, Monographs and Research Notes in Mathematics, CRC Press, Boca Raton, FL, 2016.

[3] R. M. Aron, F. J. García-Pacheco, D. Pérez-García, and J. B. Seoane-Sepúlveda, On dense-lineability of sets of functions on $\mathbb{R}$, Topology 48 (2009), no. 2-4, 149-156.

[4] R. M. Aron, V. I. Gurariy, and J. B. Seoane-Sepúlveda, Lineability and spaceability of sets of functions on $\mathbb{R}$, Proc. Amer. Math. Soc. 133 (2005), no. 3, 795-803, DOI 10.1090/S0002-9939-04-07533-1.

[5] K. Barański, On the dimension of graphs of Weierstrass-type functions with rapidly growing frequencies, Nonlinearity 25 (2012), no. 1, 193-209. 
[6] _ Dimension of graphs of Weierstrass-type functions, Fractal geometry and stochastics V, 77-91, Progr. Probab., 70, Birkhäuser/Springer, Cham, (2015).

[7] K. Barański, B. Bárány, and J. Romanowska, On the dimension of the graph of the classical Weierstrass function, Adv. Math. 265 (2014), 32-59, DOI 10.1016/j.aim.2014.07.033.

[8] A. Bartoszewicz, M. Bienias, M. Filipczak, and S. Głąb, Strong c-algebrability of strong Sierpinski-Zygmund, smooth nowhere analytic and other sets of functions, J. Math. Anal. Appl. 412 (2014), no. 2, 620-630, DOI 10.1016/j.jmaa.2013.10.075.

[9] L. Bernal-González, M. C. Calderón-Moreno, and J. A. Prado-Bassas, The set of space-filling curves: topological and algebraic structure, Linear Algebra Appl. 467 (2015), 57-74, DOI 10.1016/j.laa.2014.11.014.

[10] L. Bernal-González, D. Pellegrino, and J. B. Seoane-Sepúlveda, Linear subsets of nonlinear sets in topological vector spaces, Bull. Amer. Math. Soc. (N.S.) 51 (2014), no. 1, 71-130, DOI 10.1090/S0273-0979-2013-01421-6.

[11] D. Cariello and J. B. Seoane-Sepúlveda, Basic sequences and spaceability in $\ell_{p}$ spaces, J. Funct. Anal. 266 (2014), no. 6, 3797-3814, DOI 10.1016/j.jfa.2013.12.011.

[12] P. H. Enflo, V. I. Gurariy, and J. B. Seoane-Sepúlveda, Some results and open questions on spaceability in function spaces, Trans. Amer. Math. Soc. 366 (2014), no. 2, 611-625, DOI 10.1090/S0002-9947-2013-05747-9.

[13] K. J. Falconer, Fractal Geometry: Mathematical Foundations and Applications, Wiley \& Sons, 1990.

[14] P. Jiménez-Rodríguez, G. A. Muñoz-Fernández, and J. B. Seoane-Sepúlveda, On Weierstrass' Monsters and lineability, Bull. Belg. Math. Soc. Simon Stevin 20 (2013), no. 4, 577-586.

[15] J. L. Kaplan, J. Mallet-Paret, and J. A. Yorke, The Lyapunov dimension of a nowhere differentiable attracting torus, Ergodic Theory Dynam. Systems 4 (1984), no. 2, 261281, DOI 10.1017/S0143385700002431.

[16] G. Keller, A simpler proof for the dimension of the graph of the classical Weierstrass function, Ann. Inst. Henri Poincaré Probab. Stat. 53 (2017), no. 1, 169-181, DOI 10.1214/15-AIHP711 (English, with English and French summaries).

[17] B. B. Mandelbrot, Fractals: form, chance, and dimension, Revised edition, W. H. Freeman and Co., San Francisco, Calif., 1977. Translated from the French.

[18] R. D. Mauldin and S. C. Williams, On the Hausdorff dimension of some graphs, Trans. Amer. Math. Soc. 298 (1986), no. 2, 793-803.

[19] M. McClure, The prevalent dimension of graphs, Real Anal. Exchange 23 (1997/98), 241-246.

[20] J. B. Seoane-Sepúlveda, Chaos and lineability of pathological phenomena in analysis, ProQuest LLC, Ann Arbor, MI, 2006. Thesis (Ph.D.)-Kent State University.

[21] W. Shen, Hausdorff dimension of the graphs of the classical Weierstrass functions, Math. Z., posted on 2017, DOI https://doi.org/10.1007/s00209-017-1949-1. 


\begin{abstract}
A. BONILla
Departamento de Análisis Matemático

UNIVERSIDAD DE LA LAGUNA

C/ Astrofísico Francisco SÁnchez S/N

La Laguna (Tenerife), 38271, Spain.

Email address: abonilla@ull.es
\end{abstract}

G.A. Muñoz-Fernández

Instituto DE MATEMÁtiCA INTERDisCIPLINAR (IMI)

Departamento de Análisis y Matemática Aplicada

Facultad de Ciencias Matemáticas

Plaza de Ciencias 3

Universidad Complutense de Madrid

MADRID, 28040 (SPAIN).

Email address: gustavo_fernandez@mat.ucm.es

J.A. Prado-Bassas

Corresponding Author

Departamento de Análisis Matemático

FACUltad de Matemáticas

UNIVERSIDAD DE SEVILla

Avenida Reina Mercedes S/N

SEvilla, 41080, Spain.

Email address: bassas@us.es

J.B. SeOANE-SEPÚlVEDA

Instituto DE MATEmática InTERdisciplinar (IMI)

Departamento de Análisis y Matemática Aplicada,

Facultad de Ciencias Matemáticas

Universidad Complutense DE MADRID

Plaza de Ciencias 3

MADRID, 28040, SPAIN.

Email address: jseoane@ucm.es 